\title{
PRODUÇÃO ESPECÍFICA DE BIOGÁS A PARTIR DE MANIPUEIRA EM REATOR DE FASE ÚNICA
}

\author{
OSVALDO KUCZMAN ${ }^{1}$, SIMONE D. GOMES ${ }^{2}$, MARIA H. F. TAVARES ${ }^{3}$, \\ DOUGLAS G. B. TORRES ${ }^{4}$, MICHAEL S. ALCÂNTARA ${ }^{5}$
}

\begin{abstract}
RESUMO: O presente artigo mostra um estudo sobre a produção de biogás a partir de manipueira, considerando o volume de um reator de fluxo tubular horizontal de fase única. O objetivo do trabalho foi avaliar a produção de biogás aumentando-se as cargas orgânicas e os volumes de alimentação, o que resultou em redução do tempo de retenção hidráulica (TRH). O reator foi alimentado continuamente, com cargas orgânicas de 1,18; 1,28; 1,57 e 2,68 g DQO L ${ }^{-1} \mathrm{rd}^{-1}$ (gramas de demanda química de oxigênio por litro de reator por dia) em TRHs de 15;13,0;8,3;6,6 dias, respectivamente. As produções de biogás foram de 0,$52 ; 0,41 ; 0,65$ e $0,63 \mathrm{~L} \mathrm{~L}^{-1} \mathrm{rd}^{-1}$, destacando-se que o primeiro TRH avaliado foi o de 13 dias, seguido de 8,3; 6,6 e 15 dias. Do estudo, concluiu-se que, com a diminuição do TRH, ocorre maior produção de biogás por volume de reator. Consequentemente, através da utilização do TRH adequado, pode-se aproveitar melhor o volume do reator, fato que resultará em economia na implantação da tecnologia em indústrias processadoras de mandioca.
\end{abstract}

PALAVRAS-CHAVE: água residuária, digestão anaeróbia, fecularia, mandioca, metano.

\section{SPECIFIC BIOGAS PRODUCTION FROM MANIPUEIRA AT ONE-PHASE REACTOR}

ABSTRACT: This article presents a study on biogas production from wastewater cassava considering the volume of a one-phase horizontal tubular flow reactor. The objective was to evaluate the biogas production, enlarging the organic loadings and feeding volums, resulting in minor hydraulic retention times (HRT). The reactor was continuously fed with organic charges corresponding to $1.18 ; 1.28 ; 1.57$ and $2.68 \mathrm{~g} \mathrm{COD} \mathrm{L}^{-1} \mathrm{r} \mathrm{d}^{-1}$ (grams of oxygen chemical demand per liter of reactor per day), resulting in hydraulic retention times $15 ; 13 ; 8.3$ and 6.6 days. The correspondent specific biogas productions were, respectively, $0.52 ; 0.41 ; 0.65$ and $0.63 \mathrm{~L} \mathrm{~L}^{-1} \mathrm{rd}^{-1}$, emphasizing that the 13-day was the first HRT rated, followed by 8.3, 6.6 and 15 days. It was concluded that with the HRT decrease occurred larger biogas production per reactor volume. Therefore, with the correct selection for HRT, it is possible to make the best of the reactor volume, resulting in economy at the technology implantation in cassava starch industries.

KEYWORDS: wastewater, anaerobic digestion, cassava starch industry, cassava, methane.

\section{INTRODUÇÃO}

O Brasil produz aproximadamente 26 milhões de toneladas de mandioca, gerando cerca de 10 milhões de empregos (AGENCIA BRASIL, 2009). Com perspectivas de crescimento em todo o mundo (ZISKA et al., 2009), o segmento industrial da mandioca opera processos geradores de subprodutos com elevadas cargas orgânicas e grandes vazões (CAMPOS et al., 2006). COLIN et al. (2007) afirmam que diversas fecularias na Colômbia geram em média $11.000 \mathrm{~L}$ de água residuária para processar $1 \mathrm{t}$ de mandioca, poluindo o equivalente a 300 a 450 pessoas por dia.

\footnotetext{
${ }^{1}$ Eng $^{\mathrm{o}}$ Agrícola, doutorando em Recursos Hídricos e Meio Ambiente, UNIOESTE, bioma@ @reapr.org.br.

${ }^{2}$ Eng ${ }^{\mathrm{a}}$ Agrônoma, Profa. Doutora, UNIOESTE, simoned@unioeste.br.

${ }^{3}$ Eng ${ }^{a}$ Química, Profa., Pós-doutora, UNIOESTE, mhstavar@gmail.com.

${ }^{4}$ Eng $^{-}$Agrícola, doutorando em Recursos Hídricos e Meio ambiente, UNIOESTE, douglasgbtorres@ yahoo.com.br.

${ }^{5}$ Eng ${ }^{\mathrm{o}}$ Agrícola, mestrando em Recursos Hídricos e Meio Ambiente, UNIOESTE, eamsa_1@ hotmail.com.br.

Recebido pelo Conselho Editorial em: 12-11-2009

Aprovado pelo Conselho Editorial em: 19-11-2010
} 
Para UBALUA (2007), as lagoas são a forma mais comum de tratamento de efluente de fecularia, embora apresentem desvantagens, como emissão de gases para a atmosfera, sistemas não otimizados de decomposição anaeróbia, riscos de sobrecarga das lagoas e falta de monitoramento do processo. Consideradas como sistemas naturais para o tratamento de efluentes, as lagoas têm despertado renovado interesse (LIU, 2007).

Já segundo ANGONESE (2007), os biodigestores são tecnologias eficientes para evitar a emissão de $\mathrm{CO}_{2}$ equivalente, desde que o metano seja queimado.

Devido à possibilidade de acidificação do reator no processo de biodigestão de efluente de fecularia, vários autores salientaram a necessidade de um sistema com separação de fases para o tratamento anaeróbio da manipueira, o que pode aumentar o custo de implantação ao ser comparado com o sistema de uma fase. FEIDEN \& CEREDA (2003) recomendou a realização de pesquisas com o sistema horizontal de tratamento anaeróbio de manipueira. Dentre os poucos trabalhos com sistema de uma fase, destacam-se os trabalhos de CUZIN et al. (1992) e RIBAS \& BARANA (2003).

BÖRJESSON \& BERGLUND (2007) avaliaram o impacto ambiental total, quando sistemas de biogás são introduzidos para substituir fontes convencionais de energia, manejo de resíduos e produção agrícola. O sistema avaliado considera a combinação de materiais in natura e o uso final do biogás na produção de calor, potência e transporte de combustível.

A conclusão geral é que, normalmente, sistemas de biogás conduzem a ganhos ambientais devido ao benefício indireto do manejo do solo e a manipulação de produtos dos resíduos orgânicos, como redução da lixiviação de nitrogênio, amônia e metano.

Esses ganhos ambientais são mais relevantes do que os benefícios ambientais diretos, como substituição de combustíveis fósseis pelo biogás, que gera redução de dióxido de carbono e poluentes no ar. Segundo os autores, esses benefícios indiretos raramente são considerados, quando se avalia o biogás do ponto de vista ambiental (BÖRJESSON \& BERGLUND, 2007).

CHAVALPARIT \& ONGWANDEE (2009) citaram o uso do biogás como substituto de combustível fóssil em fecularias na Tailândia como uma opção de tecnologia limpa para o tratamento de subprodutos. MSHANDETE \& PARAWIRA (2009) atribuem ao aumento do interesse no biogás como fonte de energia a substituição de combustível fóssil que o mesmo proporciona e as vantagens ambientais por ser gerado de recursos renováveis.

GODOY \& SANTOS (2004) realizaram estudos de como as agroindústrias de mandioca se adaptam à legislação ambiental ao lançarem os efluentes em corpos receptores. A conclusão é de que as pequenas agroindústrias têm dificuldades e custos maiores em seu controle para atender à legislação, o que constitui mais uma desvantagem para as micros e pequenas empresas.

Com o objetivo de desenvolvimento de tecnologia economicamente atrativa, este artigo avalia, a partir de variações de TRH, a produção de biogás por volume de câmara de fermentação em reator horizontal de uma fase, em escala de laboratório, no tratamento da manipueira de fecularia.

\section{MATERIAL E MÉTODOS}

O reator com volume de 16,1 L foi construído no Laboratório de Saneamento da Universidade Estadual do Oeste do Paraná, Câmpus de Cascavel, com a utilização de tubos de PVC com diâmetro de $200 \mathrm{~cm}$ e comprimento de $92 \mathrm{~cm}$, sendo imerso em água para a manutenção da temperatura em $33 \pm 1{ }^{\circ} \mathrm{C}$. A inoculação foi realizada com lodo, provindo da lagoa anaeróbia da Fecularia Zadimel, localizada na latitude $24^{\circ} 41^{\prime} 19,04^{\prime \prime S}$ e longitude 53०49'58,77"W.

A adição de manipueira no reator foi iniciada com pequenos volumes diários, conforme Tabela 1, até atingir 1,25 $\mathrm{L} \mathrm{d}^{-1}$, equivalente ao TRH de 13 dias, o qual foi o primeiro TRH a ser testado. 
TABELA 1. Carga específica na partida e na operação do reator, tempo de retenção hidráulica e período de manutenção da carga. Specific load at startup and operation of the reactor, hydraulic retention time and time under load.

\begin{tabular}{|c|c|c|}
\hline $\begin{array}{c}\text { Carga Orgânica Específica } \\
\left(\mathrm{gDQO} \mathrm{L} \mathrm{r}^{-1} \mathrm{~d}^{-1}\right)\end{array}$ & $\begin{array}{c}\text { Tempo de Retenção Hidráulica } \\
\text { TRH (dias) }\end{array}$ & $\begin{array}{l}\text { Período de Manutenção da Carga } \\
\text { (dias) }\end{array}$ \\
\hline $1,12^{(1)}$ & 16,1 & 3 \\
\hline $1,27^{(1)}$ & 14,4 & 2 \\
\hline $1,34^{(1)}$ & 13,6 & 1 \\
\hline $1,41^{(1)}$ & 13,0 & 20 \\
\hline $1,29^{(2)}$ & 13,0 & 15 \\
\hline $1,62^{(2)}$ & 8,3 & 15 \\
\hline $2,70^{(2)}$ & 6,6 & 15 \\
\hline
\end{tabular}

(1) Carga orgânica na partida (2) Carga orgânica na operação

O baixo período de manutenção da carga de partida no reator, à exceção do TRH de 13 dias, deveu-se ao inóculo ocupar todo o volume do reator. A manutenção da partida durante 20 dias, para o TRH de 13 dias, ocorreu devido à necessidade de se aguardar, pelo menos, um tempo de 13 dias para eliminar o efeito das cargas de partida no TRH a ser estudado. $\mathrm{O}$ acompanhamento da aclimatação dos microrganismos foi realizado através do monitoramento dos valores da relação Acidez Volátil (AV)/Alcalinidade Total (AT), da DQO, do pH e da produção de biogás. A relação AV/AT manteve-se, em média, entre 0,14 e 0,30, ressaltando que a manipueira utilizada, em todo o experimento, foi adicionada como coletada na indústria, sem adição de nutrientes ou neutralizantes.

Para compensar a fração de água evaporada devido ao aquecimento, retirou-se a manipueira fermentada na saída do reator, diariamente, por sucção, sendo o volume aferido através do controle da altura da coluna de efluente em fermentação. $\mathrm{O}$ volume diário de manipueira correspondente ao TRH em estudo era armazenado em recipiente plástico e transferido ao reator diariamente por bomba peristáltica elétrica. Para diminuir o TRH, aumentava-se gradativamente o volume de alimentação e, ao atingir o TRH a ser avaliado, aguardava-se o equivalente a 15 dias, para eliminar a interferência do TRH anterior. A medição do biogás foi realizada através da determinação do volume de solução acidossalina, com densidade igual a $1,173 \mathrm{~g} \mathrm{~cm}^{-3}$, deslocada pela pressão interna do reator. Na Figura 1, mostra-se o esquema da montagem do reator.

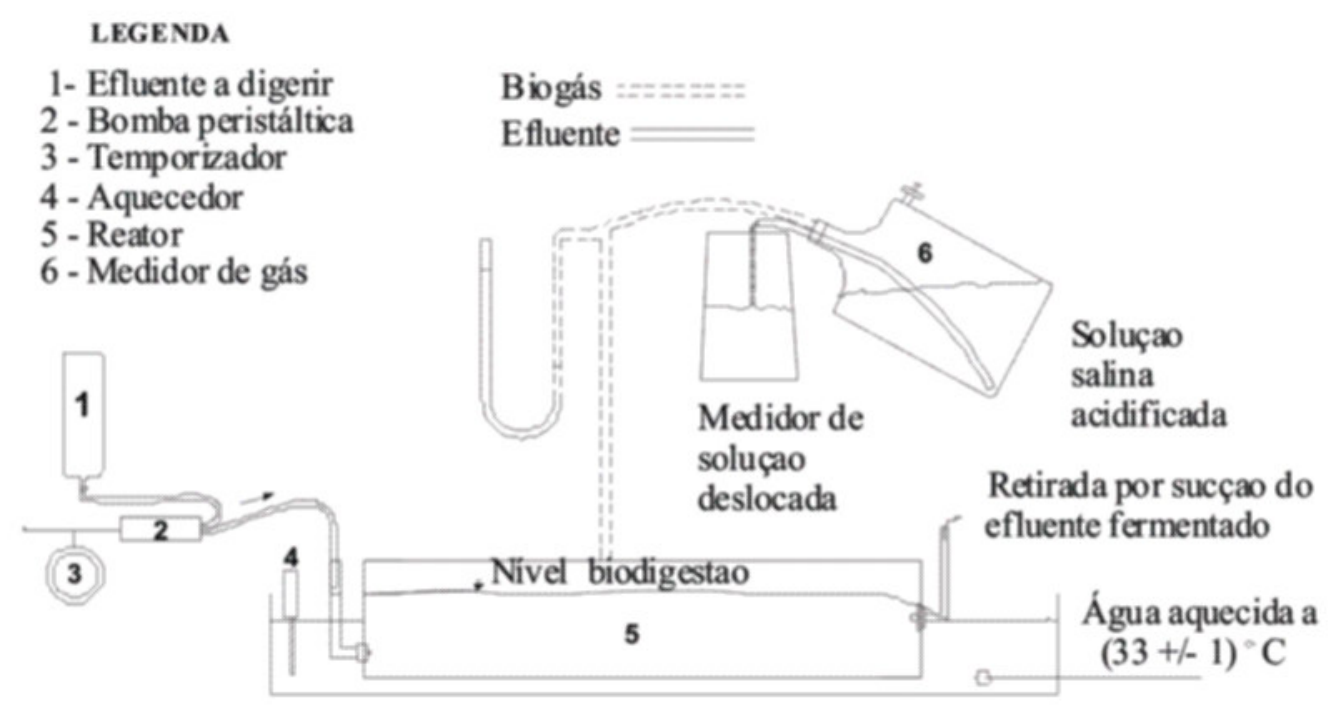

FIGURA 1. Esquema da montagem do reator. Reactor scheme.

A pressão manométrica no reator foi transformada em $\mathrm{mm}$ de $\mathrm{Hg}$ pela eq.(1):

$\mathrm{P}_{\mathrm{r}}=1,359 \quad \mathrm{P}_{\mathrm{m}} 1,173$ 
em que,

$\mathrm{P}_{\mathrm{r}}$ - pressão manométrica no reator $(\mathrm{mm} \mathrm{Hg})$;

$\mathrm{P}_{\mathrm{m}}$ - pressão no manômetro (centímetros de coluna de solução acidossalina), e

1,173 - fator de conversão de comprimento de coluna de solução acidossalina para comprimento de coluna de água.

Na sequência, o valor de $P_{r}$ foi substituído na eq.(2):

$$
V=\frac{273}{273+t} \frac{P+P_{r}}{760} V_{d} F
$$

em que,

$\mathrm{V}$ - volume de biogás expresso em $\mathrm{CNTP}_{(\mathrm{seco})}$;

$\mathrm{V}_{\mathrm{d}}$ - volume de biogás avaliado pelo deslocamento da solução;

$\mathrm{F}$ - fator de correção da umidade à temperatura do local do experimento

$\mathrm{P}$ - pressão local em mm $\mathrm{Hg}$, e

$\mathrm{t}$ - temperatura local do experimento em ${ }^{\circ} \mathrm{C}$;

As análises químicas foram realizadas de acordo com APHA (1998). A análise estatística foi efetuada através do programa MINITAB 15 e constou de análise de variância com o modelo múltiplo com variáveis dummy ou artificiais e de regressão, de comparação de médias, pelo método de Tukey, a 5\% de significância.

\section{RESULTADOS E DISCUSSÕES}

Na Tabela 2, apresenta-se a composição média da manipueira utilizada no experimento.

TABELA 2. Composição média da manipueira. Average composition of the manipueira

\begin{tabular}{lc}
\hline \multicolumn{1}{c}{ Característica } & Valor \\
\hline \multicolumn{1}{c}{$\mathrm{pH}$} & 4,37 \\
Demanda Química de Oxigênio (DQO) & $15,72 \mathrm{~g} \mathrm{~L}^{-1}$ \\
Sólidos Totais (ST) & $9,54 \mathrm{~g} \mathrm{~L}^{-1}$ \\
Sólidos Voláteis (SV) & $7,51 \mathrm{~g} \mathrm{~L}^{-1}$ \\
Sólidos Fixos (SF) & $2,03 \mathrm{~g} \mathrm{~L}^{-1}$ \\
\hline
\end{tabular}

Os valores médios das produções diárias específica de biogás são apresentados na Tabela 3.

TABELA 3. Comparação das médias de produção diária específica de biogás. Comparison of biogas specific production average values.

\begin{tabular}{cc}
\hline TRH $(\mathrm{d})$ & Produção Específica de Biogás $\left(\mathrm{L} \mathrm{gás} \mathrm{L}^{-1} \mathrm{r} \mathrm{d}^{-1}\right)$ \\
\hline 15,0 & $0,52 \mathrm{bc}$ \\
13,0 & $0,41 \mathrm{c}$ \\
8,3 & $0,65 \mathrm{a}$ \\
6,6 & $0,63 \mathrm{ab}$ \\
\hline
\end{tabular}

Letras minúsculas iguais correspondem a médias iguais, a 5\% de probabilidade, pelo teste de Tukey.

Com a diminuição do TRH, houve aumento nas produções de biogás. A ordem de condução do experimento, primeiramente realizando-se o TRH de 13 dias, seguido de 8,3 e 6,6 e só então o TRH de 15 dias, pode explicar o pequeno ganho em produção de biogás verificada entre o TRH de 15 e 6,6 dias, $21 \%$, quando comparada com o ganho entre o TRH de 13 e 6,6 dias que apresentou aumento de $54 \%$. A alteração da ordem pode ter levado à quebra no equilíbrio dos diversos grupos de microrganismos atuantes na digestão anaeróbia, estabelecendo a igualdade estatística na 
produção de biogás entre os TRHs de 15 e 6,6 dias. Através da Figura 2, pode-se verificar a tendência de aumento na produção de biogás com a redução do TRH.

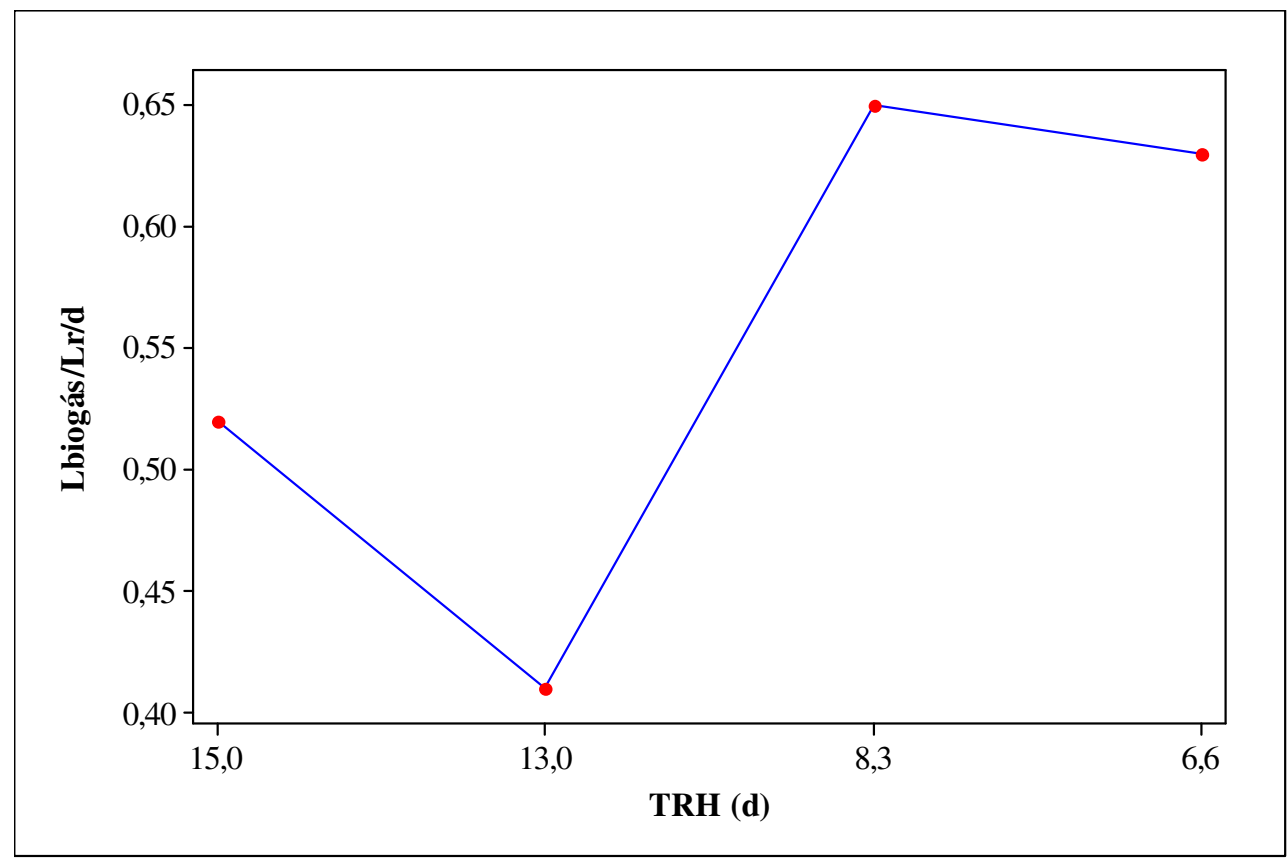

FIGURA 2. Variação da produção de biogás com a redução do TRH. Biogas production variation with HRT reduction.

CHAIPRASERT et al. (2003) verificaram a mesma tendência ao tratarem manipueira suplementada de minerais com carga orgânica de $4 \mathrm{~g}$ DQO L ${ }^{-1} \mathrm{r} \mathrm{d}^{-1}$, utilizando reatores híbridos de fluxo ascendente em uma fase com fibras de náilon para meio suporte. Para os TRHs de 18,2; 9,3; 6,2 e 5,4, as produções específicas de biogás foram de 0,$84 ; 1,59 ; 4,23$ e $5,54 \mathrm{~L} \mathrm{~L}^{-1} \mathrm{r} \mathrm{d}^{-1}$, respectivamente, o que representa aumento de $560 \%$ entre os TRHs de 18,2 e 5,4 dias.

NAJAFPOUR et al. (2006) operaram em laboratório um reator de fluxo ascendente com leito de lodo e meio suporte alimentado com efluente da extração industrial de óleo de palma e obtiveram aumento médio de $8 \%$ na produção de biogás ao se reduzir o TRH de 3 para 1,5 dia.

COLIN et al. (2007) experimentaram o efeito do bambu para suporte de microrganismos em reator filtro de fluxo horizontal na fermentação de manipueira de fecularia produtora de polvilho (fécula) azedo. Nos TRHs de 43,2; 20,5; 13,4; 9,2 e 9,6 dias, as produções específicas de biogás foram de 0,$52 ; 0,81 ; 1,72 ; 2,13$ e $2,65 \mathrm{~L} \mathrm{~L}^{-1} \mathrm{r} \mathrm{d}^{-1}$,com aumento de $410 \%$ entre os TRHs de $43,2 \mathrm{e}$ 9,6 dias.

As pesquisas confirmam a tendência de aumento na produção de biogás por volume de reator com a diminuição do TRH, que pode ser explicado pelo aumento no metabolismo dos microrganismos promotores da digestão anaeróbia diante da maior oferta de substrato.

O teste apresentado na Tabela 4 mostra a significância das variáveis explicativas na análise de regressão dos dados da produção de biogás por volume de reator. Neste modelo, o tempo não é significativo ao nível de 5\% de significância, permitindo concluir que, cada medida diária nos quinze dias avaliados, pode ser considerada como repetição nos tratamentos adotados, tendo sido ajustado o seguinte modelo:

Biogás por volume de reator $=0,536-0,00225$ Tempo $(\mathrm{d})-0,104 \mathrm{Z1}+0,137 \mathrm{Z2}+0,110 \mathrm{Z3}$ 
TABELA 4. Teste de significância das variáveis explicativas na análise de regressão de produção de biogás por volume de reator por dia. Significance test of the explicative variables at the regression analysis for daily specific biogas production.

\begin{tabular}{cccccc}
\hline Variáveis Explicativas & Coeficiente & Desvio-Padrão & Estatística t & P - valor & Decisão \\
\hline Constante & 0,53555 & 0,04457 & 12,02 & 0,000 & $*$ \\
Tempo (d) & $-0,00225$ & 0,00379 & $-0,60$ & 0,554 & NS \\
Z1 & $-0,10400$ & 0,04625 & $-2,25$ & 0,029 & $*$ \\
Z2 & 0,13680 & 0,04625 & 2,96 & 0,005 & $*$ \\
Z3 & 0,10980 & 0,04625 & 2,37 & 0,021 & $*$ \\
\hline
\end{tabular}

NS - não significativa a 5\% de significância. * - significativa a 5\% de significância.

Na Tabela 5, apresentam-se os resultados da análise de variância para avaliar o modelo ajustado, para produção de biogás por volume de reator. Como $\mathrm{P}<0,05$, pelo menos uma das variáveis explicativas afetou a variável resposta.

TABELA 5. Análise de variância do modelo ajustado para produção de biogás por volume de reator. Adjusted model analysis of variance for daily specific biogas production.

\begin{tabular}{cccccc}
\hline Fonte de & Graus de & Soma & Quadrado & \multirow{2}{*}{ Estatística F } & \multirow{2}{*}{ Valor de P } \\
Variação & Liberdade & Quadrada & Médio & & $0,000^{*}$ \\
\hline Regressão & 4 & 0,55322 & 0,13831 & 8,62 & \\
Resíduo & 55 & 0,88239 & 0,01604 & & \\
Total & 59 & 1,43562 & & & \\
\hline
\end{tabular}

Na Tabela 6, apresentam-se as médias da relação acidez volátil versus alcalinidade total e de demanda química de oxigênio de entrada e saída do reator nos tratamentos adotados.

TABELA 6. Resultados de relação AV/AT e DQOs afluente e efluente do reator. Results of AV / AT relation and COD's affluent and effluent of reactor.

\begin{tabular}{cccc}
\hline TRH (dias) & AV/AT & DQO Afluente $\left(\mathrm{g} \mathrm{L}^{-1}\right)$ & DQO Efluente $\left(\mathrm{g} \mathrm{L}^{-1}\right)$ \\
\hline 15 & 0,14 & 17,65 & 0,52 \\
13 & 0,30 & 16,52 & 0,13 \\
8,3 & 0,19 & 13,31 & 0,57 \\
6,6 & 0,27 & 17,65 & 0,83 \\
\hline
\end{tabular}

\section{CONCLUSÕES}

Em reatores anaeróbios operados com manipueira, a produção de biogás por volume de reator é aumentada, até determinado limite, ao se reduzir o tempo de retenção hidráulica da fermentação.

Ao se diminuir o TRH de 15 para 6,6 dias, a produção de biogás passou de 0,52 para $0,63 \mathrm{~L}_{\text {gás }} \mathrm{L}^{-1} \mathrm{r} \mathrm{d}^{-1}$, ou seja, ocorreu aumento de $21 \%$.

A redução do TRH implica diminuição do tamanho do reator, tornando sua construção mais econômica.

\section{REFERÊNCIAS}

AGÊNCIA BRASIL. Disponível em:

http://www.agenciabrasil.gov.br/noticias/2009/02/27/materia.2009-02-27.8598137216/view>.

Acesso em: 28 ago. 2009.

ANGONESE, A.R.; CAMPOS, A.T.; WELTER, R.A. Potencial de redução de emissão de equivalente de carbono de uma unidade suinícola com biodigestor. Engenharia Agrícola, Jaboticabal, v.27, n.3, p.648-657, 2007. 
APHA. Standard methods for the examination of water and wastewater. $20^{\text {th }}$ ed. Washington: American Public Health Association, 1998. 1.268 p.

BÖRJESSON, P.; BERGLUND, M. Environmental systems analysis of biogas systems - Part II: The environmental impact $\mathrm{f}$ replacing various reference systems. Biomass and Bioenergy, Oxford, v.21, n.2, p.326-344, 2007.

CAMPOS, A.T.; DAGA, J.; RODRIGUES, E.E.; FRANZENER, G.; SUGUY, M.M.; SYPERRECK, V.I.G. Tratamento de águas residuárias de fecularia por meio de lagoas de estabilização. Engenharia Agrícola, Jaboticabal, v.26, n.1, p.235-242, 2006.

CHAIPRASERT, P.; SUVAJITTANONT, W.; SURARAKSA, B.; TANTICHAROEN, M.; BHUMIRATANA, S. Nylon fibers as supporting media in anaerobic hybrid reactors: it's effects on system's performance and microbial distribution. Water Research, Oxford, v.37, n.19, p.4.6054.612, 2003.

CHAVALPARIT, O.; ONGWANDEE, M. Clean technology for the tapioca starch industry in Thailand. Journal of Cleaner Production, Amsterdam, v.17, n.2, p.105-110, 2009.

COLIN, X.; FARINET, J.L.; ROJAS, O.; ALAZARD, D. Anaerobic treatment starch of cassava extraction wastewater using a horizontal flow filter with bamboo as support. Bioresource Technology, Amsterdam, v.98, n.8, p.1.602-1.607, 2007.

CUZIN, N.; FARINET, J. L.; SEGRETAIN, C.; LABAT, M. Methanogenic fermentation of cassava peel using a pilot plug flow digester. Bioresource Technology, Amsterdam, v.41, n.3, p.259-264, 1992.

FEIDEN, A.; CEREDA, M.P. Potencial energético do biogás gerado no tratamento de águas residuárias de fecularias em sistema piloto de biodigestão anaeróbia com separação de fases. Energia na Agricultura, Botucatu, v.18, n.2, p.54-66, 2003.

GODOY, A.M.G.; SANTOS, R.J.C. Gestão dos efluentes líquidos das indústrias de farinha de mandioca de Araruna - PR. Produto \& Produção, Porto Alegre, v.7, n.3, p.37-49, 2004.

LIU, S. Food and agricultural wastewater utilization and treatment. Ames: Blackwell Publishing, 2007. 277 p.

MINITAB. Minitab for Windows - version 15.0. Pennsylvania: State College, 2008.

MSHANDETE, A. M.; PARAWIRA W. Biogas technology research in selected sub-Saharan African countries - A review. African Journal of Biotechnology, Abraka, v.8, n.2, p.116-125, 2009.

NAJAFPOUR, G.D.; ZINATIZADEH, A.A.L.; MOHAMED, A.R.; HASNAIN ISA, M.; NASROLLAHZADEH, H. High-rate anaerobic digestion of palm oil mill effluent in an upflow anaerobic sludge-fixed film bioreactor. Process Biochemistry, London, v.41, n.2, p.370-379, 2006.

RIBAS, M.M.F.; BARANA, A.C. Start-up Adjustment of a plug-flow digester for cassava wastewater (manipueira) treatment. Scientia Agrícola, Piracicaba, v.60, n.2, p.223-229, 2003.

UBALUA, A. O. Cassava wastes: treatment options and value addition alternatives. African Journal of Biotechnology, Abraka, v.6, n.18, p.2.065-2.073, 2007.

ZISKA, L.H.; RUNION, G.B.; TOMECEK, M.; PRIOR, S.A.; TORBET, H.A.; SICHER, R. An evaluation of cassava, sweet potato and field corn as potential carbohydrate sources for bioethanol production in Alabama and Maryland. Biomass and Bioenergy, Oxford, v.33, n.11, p.1.503-1.508, 2009. 